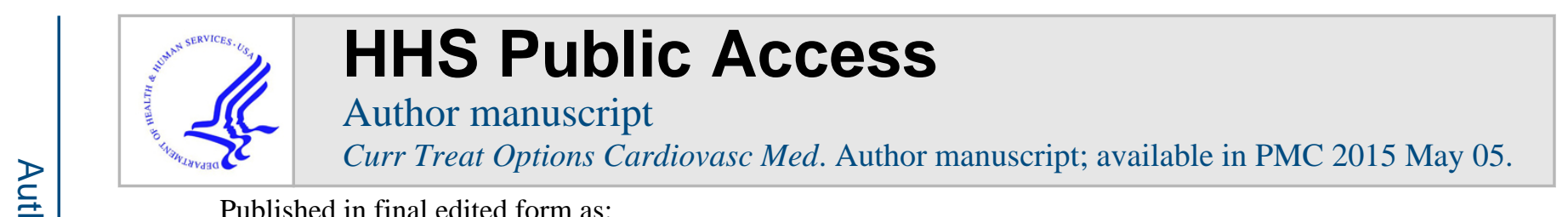

Published in final edited form as:

Curr Treat Options Cardiovasc Med. 2015 January ; 17(1): 358. doi:10.1007/s11936-014-0358-6.

\title{
Controversies in Cardioembolic Stroke
}

\author{
Benjamin S. Wessler, MD ${ }^{1,2}$ and David M Kent, MD, MS ${ }^{1}$ \\ ${ }^{1}$ Predictive Analytics and Comparative Effectiveness (PACE) Center, Institute for Clinical \\ Research and Health Policy Studies (ICRHPS), Tufts Medical Center/Tufts University School of \\ Medicine, Boston \\ ${ }^{2}$ Division of Cardiology, Tufts Medical Center, Boston
}

\section{Opinion statement}

Cardioembolic (CE) stroke mechanisms account for a significant number of ischemic strokes however the true burden is likely underestimated. It is critically important to identify patients with $\mathrm{CE}$ strokes since these individuals have high recurrence rates and represent a subgroup of patients who may benefit from targeted therapy in the form of anticoagulation or device based treatments. Current guidelines offer recommendations for diagnosis and treatment of these patients however important questions remain. Appropriate cardiac testing in the setting of CE must be individualized and the optimal duration of electrocardiographic monitoring to rule out atrial fibrillation (AF) is unclear. So too, risk stratification tools for AF remain understudied and there is controversy about which anticoagulant agents are most appropriate. Lastly, important potential CE sources of stroke such as PFO have garnered significant attention recently and debate regarding how to treat these patients remains. In this review we discuss some of the important controversies in diagnosing and treating patients with possible $\mathrm{CE}$ stroke, pointing to areas where future research might be particularly valuable.

\section{Keywords}

cardioembolic stroke; ischemic stroke; atrial fibrillation; patent foramen ovale; cryptogenic stroke; anticoagulation

\section{Introduction}

Despite decreasing stroke incidence and lower case fatality rates over the last century, stroke syndromes remain a significant cause of morbidity and mortality. Stroke represents the second most common cause of death and the third most common cause of disability worldwide.[1,2] Approximately 6.8 million American adults $\geq 20$ years of age have had a stroke and there are approximately 800,000 events annually of which $87 \%$ are ischemic.[3] For individual patients with ischemic stroke the natural history, risk of recurrence, and treatment strategies all vary widely based on the presumed stroke mechanism.

Corresponding Author: David M Kent, MD, CM, MSc, Predictive Analytics and Comparative Effectiveness (PACE) Center, Institute for Clinical Research \& Health Policy Studies (ICRHPS), Tufts Medical Center, 800 Washington St, Box 63, Boston, MA, 02111, Phone: (617) 636-3234, Fax: (617) 636-0022, Dkent1@ tuftsmedicalcenter.org. 
Cardioembolic (CE) causes of stroke account for up to $20 \%$ of ischemic strokes and represent a subgroup of strokes with a high rate of recurrence. These patients have a clear potential therapeutic target (anticoagulation or device based treatments) however they continue to experience significant morbidity and mortality.[4] The pathophysiologic causes of CE strokes are highly varied and can be categorized as originating from cardiac lesions that tend to form thrombus (mechanical valves, left atrial appendage [LAA]), cardiac masses (i.e. tumors, vegetations, etc), or sources of paradoxical embolism from venous thrombosis (e.g. patent foramen ovale [PFO]).[5] Despite the clear importance of identifying and treating $\mathrm{CE}$ sources of ischemic stroke, many questions for patients and clinicians still exist. In this review we identify some of the major controversies in the evaluation and management of patients with CE stroke and discuss the recent clinical trial data that inform these debates.

\section{Controversy 1: Identifying the CE Mechanism}

For individuals presenting with ischemic stroke, depressed level of conciousness, rapid symptom improvement, early onset of maximal deficit, and sequential strokes in different arterial territories all increase the likelihood that an ischemic stroke is related to a $\mathrm{CE}$ source.[6] In addition to cerebral and large vessel imaging patients should have routine testing to evaluate potential arrhythmic causes of stroke. Current guidelines recommend that patients have a 12 lead electrocardiogram and telemetry monitoring for 24 hours to identify arrhythmic causes of stroke.[7] The extent of additional evaluation for potential CE sources of stroke is highly variable and many questions remain.

\section{What Cardiac Imaging is Necessary?}

When considering CE sources of stroke it is important to perform a focused evaluation to identify potentially important cardiac lesions. The European Society of Echocardiography released guidelines to help improve the appropriateness of echocardiographic imaging in the setting of ischemic stroke.[8] These recommendations are guided by the clinical history and the stroke mechanism considered most likely for an individual. In general echocardiographic imaging is recommended when this information will alter prognosis or treatment. When evaluating lesions that are prone to thrombus formation the preferred test depends on the pathologic site. The majority of left ventricle (LV) thrombi can be effectively seen with transthoracic echocardiography (TTE) and are most often seen following myocardial infarction or in the setting of heart failure with a reduced LV ejection fraction. In contrast thrombus detection in the LAA in the setting of atrial fibrillation (AF) is often missed with TTE and transesophageal echocardiography (TEE) is needed.[9] Masses are often seen with TTE, though thorough characterization is often found only with TEE or MRI. TEE is also recommended when evaluating aortic atherosclerosis severity and complexity though TTE may offer some complimentary views. Given the non-invasive nature of TTE it is commonplace and appropriate to begin with this test when considering CE sources of stroke and only move to TEE when clinical questions remain. Ordering clinicians should be mindful that TEE requires sedation and passage of the imaging probe past the posterior oropharanyx and into the esophagus (and into the stomach for a complete exam). Complications are rare, ranging from $0.18-2.8 \%$, but include bleeding, esophageal 
perforation, hoarseness, lip or dental injury, or worsening of a variety of cardiovascular conditions. Death has also been reported.[10]

TEE has long been considered the gold standard when evaluating the presence and characteristics of a PFO in the setting of stroke.[11] This imaging technique allows for direct visualization of the PFO including size, location, severity of right to left shunting (RLSh) and also presence or absence of atrial septal aneurysms, though the significance of these so called 'high risk' features has been called into question.[12] When closure of a PFO is considered, TEE remains an essential tool for detailed evaluation of PFO. Recent data have renewed interest in transcranial Doppler (TCD) imaging as a non-invasive and highly sensitive tool for identifying RLSh. The sensitivity of this technique is likely higher than that seen with TEE and while some of the RLSh identified with TCD may be related to extracardiac shunting this technique is becoming an important non-invasive tool that can effectively rule out PFO and may have prognostic significance.[13,14] Complete evaluation of PFO likely will involve multiple imaging modalities as we move toward fully understanding the significance of this frequently encountered anatomic feature in the setting of stroke.

\section{The Difficulty with Atrial Fibrillation}

Atrial fibrillation (AF) leads to stroke via thrombus formation, most often in the LAA. AF is a major CE cause of stroke and is a major contributor to the global ischemic stroke burden. A population study suggests 1 in 6 ischemic strokes are due to this arrhythmia.[15] Identifying AF remains essential because secondary stroke prevention with oral anticoagulation vs. antiplatelet therapy leads to a 39\% relative risk reduction (RRR) of recurrent stroke (NNT $=24$ over 1 year to prevent one stroke).[16] Data on the burden of atrial fibrillation and its association with stroke, however, is based on incident arrhythmia detection at the time of an index stroke and as a result the number of strokes attributable to atrial fibrillation is likely underestimated given the paroxysmal and often asymptomatic nature of this arrhythmia.[17] Interestingly, recent evaluations suggest that even these asymptomatic episodes of atrial fibrillation are clinically important.[18] The ASSERT study enrolled 2580 patients with either pacemakers or defibrillators with no history of AF. Patients were monitored for 3 months for arrhythmia detection and 2.5 years for development of ischemic stroke or systemic embolism. $10 \%$ of the patients had subclinical atrial tachyarrhythmias. These tachyarrhythmias were associated with clinical AF (HR 5.56; 95\% confidence interval [CI], 3.78-8.17) and the composite of ischemic stroke or systemic embolic event (HR 2.49; 95\% CI, 1.28-4.85). While this study demonstrated that these subclinical arrhythmic events are associated with stroke there is no clear data that treating these patients with oral anticoagulants will improve outcomes.

There is wide practice variation in the intensity of AF detection efforts in the setting of stroke. Methods for AF detection include: history; 12 lead electrocardiography; hospital telemetry; holter monitoring, external loop recorder and implantable loop recorder. Further controversy exists about the length of monitoring that is necessary to identify AF following stroke in patients without another identified cause; data from two recent randomized controlled trials (RCTs) renewed this debate. In CRYSTAL AF, 441 patients diagnosed with 
cryptogenic stroke (CS) were randomized to either an insertable cardiac monitor (ICM) or routine follow up for detection of AF.[19] The ICM used in this study (REVEAL XT, Medtronic) was a leadless monitoring device implanted subcutaneously that allowed for long term follow up.[20] After 6 months, AF was detected in $8.9 \%$ of patients in the ICM group compared with $1.4 \%$ in the control group (HR 6.45, 95\% CI, 1.9-21.7). The EMBRACE investigators randomized 572 patients with cryptogenic stroke or TIA to 30 day event monitoring (treatment) vs. 24 hour monitor (control) for detection of onset of AF within 90 days.[21] The event monitor (ER910AF Cardiac Event Monitor, Braemar) was attached with a nonadhesive belt worn around the chest and automatically recorded AF based on an established algorithm.[22] The primary outcome (detection of AF > 30 seconds) was detected in $16.1 \%$ of patients in the treatment group compared with $3.2 \%$ in the control group representing a number needed to screen of 8 over 30 days to identify an incident case of $\mathrm{AF}$.

These trials raise important questions for clinicians treating patients with possible CE stroke. What is the duration of monitoring necessary to 'exclude' AF and what burden of AF is clinically important? Furthermore how invasive will patients and clinicians be as they work to exclude this arrhythmia and at what point is extended monitoring no longer cost effective? We don't yet know what to do for these patients with respect to oral anticoagulation though the majority of clinicians will presume that if any AF is identified in the post stroke setting and there are no contraindications, patients should be offered anticoagulation therapy. Such an approach is probably reasonable given the mechanistic uncertainty that is likely to remain. While it is clear that long term ambulatory monitoring can identify AF for a group of patients previously diagnosed with CS we do not know for an individual what AF burden is clinically import, whether an index stroke is attributable to $\mathrm{AF}$, or whether recurrent stroke risk can be minimized with anticoagulation.

\section{Controversy 2: Risk stratification for CE Stroke}

For patient who have had or are at risk for having CE stroke due to non-valvular AF the risk of recurrent or incident stroke is related to patient specific factors and decisions about oral anticoagulation are individualized. Clinicians have used the CHADS2 risk stratification tool to guide decisions about anticoagulation and in an external validation cohort of registry data from Medicare beneficiaries this clinical prediction model showed excellent discrimination with a $c$-statistic 0.82 (95\% CI, 0.80-0.84).[23] Subsequent analyses have demonstrated that the $c$-statistic ranges from $0.52-0.82$ dependent on the population under study.[24] This score, however, placed a significant number of patients (27\% in the original external validation cohort) into an intermediate risk category and as a result did not effectively identify those individuals at lowest risk for thromboembolic complications.[25] This observation and recognition that CHADS2 did not account for other important known stroke risk factors led to the recent adoption of a newer CPM.

\section{Are We Using an Improved Predictive Model?}

To address these issues the $\mathrm{CHA}_{2} \mathrm{DS}_{2} \mathrm{VASc}$ score was developed and was recently recommended as the preferred CPM for individualizing anticoagulation decisions for individuals. This newer score incorporates female sex, history of vascular disease, and 
increases the significance of age $>75$ and has replaced the CHADS2 score as the currently recommended prediction model for helping to guide decisions about anticoagulation.[25,26] The $\mathrm{CHA}_{2} \mathrm{DS}_{2} \mathrm{VASc}$ score was developed by refining the previously reported 2006 Birmingham/NICE stroke risk stratification schema. New risk factors were added and the model was applied to the Euro Heart Survey for AF, a database of 5,333 patients with AF recruited from both ambulatory and hospital sites in 35 countries in 2003-2004 and followed for 1 year. This model showed a $c$-statistic in this validation cohort of 0.61 (95\% CI, $0.51-$ 0.70 ) and classified only $9.2 \%$ of patients as low risk (with a $0 \%$ thromboembolism rate at 1 year).[26] The $c$-statistic for the $\mathrm{CHA}_{2} \mathrm{DS}_{2} \mathrm{VASc}$ score has ranged from $0.52-0.89$ as it has been studied across a variety of populations and since this CPM has proved better able to differentiate the low and intermediate risk patients it is now the preferred tool for guiding decisions about anticoagulation.[24,25] Use of this score has increased the number of people who are anticoagulated and largely redistributes older women from low to high risk categories.[27] It remains unclear, however, whether this risk redistribution will result in fewer thromboembolic events and how bleeding rates will change in contemporary clinical practice, especially with the increasing use of novel oral anticoagulants (NOACs).

Additional questions remain when assessing the performance and thus utility of this clinical prediction model (CPM). Effective discrimination is a necessary but not sufficient feature of CPM to be valuable tools for clinicians and researchers.[28] Model discrimination is a measure of how well a CPM can separate patients with the outcome of interest (in this case stroke) from those without. It is a rank order statistic and as such is insensitive to whether or not the predicted values match observed rates of disease in the populations the tool is applied, which is the relevant objective for clinical decision making.[29] To address this issue, it is essential that CPMs are assessed for calibration on contemporary validation cohorts. $\mathrm{CHA}_{2} \mathrm{DS}_{2} \mathrm{VASc}$ underwent external validation with a cohort from 2003-2004 who were not using oral anticoagulation. It is crucial to appropriately calibrate models guiding therapy for stroke prevention where the harms of anticoagulation (i.e. bleeding) must be weighed against the true rates of stroke in the population, especially considering the national trend towards fewer and less severe strokes over time.[2] A recent systematic review and meta-analysis reported that calibration data are lacking and unreliably reported for the $\mathrm{CHA}_{2} \mathrm{DS}_{2} \mathrm{VASc}$ score[24] and as a result accurate recommendations about treatment thresholds remain unclear.

\section{Controversy 3: Alternatives to Warfarin for CE Stroke Prevention}

There has been significant progress and study of alternatives to warfarin for patients with AF. Researchers have studied mechanical occlusion of the LAA to decrease the likelihood of thromboembolic complications. The success of these techniques, however, has been limited by device related complications.[30] Development and study of NOACs has expanded in recent years in response to the narrow therapeutic window and frequent dose adjustments needed to safely manage patients on warfarin. The first such agent, dabigatran, is a direct thrombin inhibitor that was studied in the RE-LY trial.[31] This RCT compared dabigatran to warfarin in 18,113 patients recruited from 44 countries around the world and demonstrated that dabigatran was associated with similar rates of the primary endpoint of stroke or systemic embolism as warfarin after 2 years of follow up. Rivaroxaban, a factor Xa 
inhibitor, was evaluated in the ROCKET AF trial in which 14,264 patients were randomized to rivaroxaban or warfarin.[32] Rivaroxaban was non-inferior to warfarin for preventing the primary endpoint, a composite of stroke (ischemic or hemorrhagic). The final NOAC available for stroke prevention in AF, the factor Xa inhibitor apixaban, was studied in the ARISTOTLE trial.[33] This trial randomized 18,201 patients with atrial fibrillation and one stroke risk factor to receive either warfarin or apixaban. Investigators followed patients for 1.8 years and found that this agent was noninferior to warfarin for prevention of the composite outcome of stroke or systemic embolism. Important secondary endpoints of death from any cause, major bleeding, and hemorrhagic stroke were all lower in the apixaban arm suggesting a possible advantage over warfarin. Emerging data suggest that NOAC therapy results in lower rates of stroke when compared to warfarin with an overall improved safety profile (Figure 1).[34] With the proliferation of these novel agents there is a noticeable lack of direct comparison, and as additional agents are added to this group[35], there is little guidance to help physicians know which agent is best for their patients.

With the development of these innovative therapeutics important questions arise. Since these agents are new and have not been used for extended periods of time, the long term risks are largely unknown. Reports of increased rates of acute coronary syndrome with dabigatran have drawn interest.[36] So too possible increased rates of thromboembolic events when rivaroxaban is stopped abruptly without bridging therapy led to a black box warning though the significance of this observation is not clear.[37] Hepatic failure with rivaroxaban was also recently described.[38] While the bleeding risks with these agents were low in the seminal clinical trials above the event rates in clinical practice, where patients are outside of the highly monitored clinical trial environment, have not yet emerged. Understanding these rates will be important for patients and providers since no effective reversal agents are yet commercially available.[39] Lastly, as is common for new technologies, the cost effectiveness of these agents must be fully evaluated to understand whether they represent a good value for our health care system.[40] While these questions remain unanswered and additional information accumulates, the most prudent approach may be to pause before embracing these novel agents and to maintain warfarin for patients who tolerate this medication well.

\section{Controversy 4: Treatment Strategies: The case of Cryptogenic stroke and PFO}

Patent Foramen Ovale (PFO) is associated with CS and it is frequently considered as a possible CE source since it allows for potential paradoxical embolism, a systemic arterial embolus from a venous source.[41,42] While this anatomic feature is present in approximately $25 \%$ of the general population, it is more commonly seen in patients with CS suggesting a causative role.[43] There has been significant interest in closing identified PFO for patients with CS in the hopes of altering the risk of stroke recurrence. Three recent trials testing different interventional devices, have all missed their primary intention to treat outcomes.[44-46] CLOSURE I randomized 909 patients to closure with the STARFlex device vs. medical therapy and followed them for 2 years. The RESPECT and PC Trials were both randomized controlled trials studying device closure with the Amplatzer PFO 
Occluder device vs. medical therapy. The RESPECT trial enrolled 980 patients and followed them for a median of 2.1 years. PC Trial randomized 414 patients and followed them an average of 4.1 years. Efforts to combine these trials through meta-analysis have not identified a clear answer as to whether or not closure is an effective therapy (Figure 2).[47] Despite the completion of 3 randomized trials with crudely consistent overall results (i.e. their summary effect measure was null), because the outcome rate is very low among medically treated patients, the power of these trials—even when combined-is limited to detect moderate effects, and many interpret the trials (particular those testing the Amplatzer device) as suggestive. These trials together raise more questions than they provide answers; it is clear that PFO closure is not clearly beneficial for everyone.[48]

Importantly, optimal medical therapy for patients with stroke and PFO has not been defined. The major trials discussed above have allowed a variety of antiplatelet and anticoagulant regimes. Definitive head-to-head studies comparing antiplatelet therapy to anticoagulation have not been performed. With the exception of patients with atrial fibrillation, known thrombi, and mechanical heart valves, where oral anticoagulation is the preferred antithrombotic, guideline-recommended care for ischemic stroke patients generally includes antiplatelet therapy.[49] However, there is considerable disagreement over the best antithrombotic approach in patients with CS and PFO. Although the clinical syndrome caused by paradoxical embolism is arterial occlusion, the thrombus arises from a venous source, and therefore response to therapy may be more analogous to that of venous thromboembolism where anticoagulation is far superior. There has been renewed interest in the optimal medical therapy of these strokes with the advent of NOACs and clinical trials are ongoing.[50] Classification of patients with CS and PFO into a broader (more heterogeneous) category of patients with "embolic stroke of undetermined source" (ESUS) may permit an average treatment effect to be determined and help clarify guidelines for the treatment of this broader group, while still leaving the question of PFO-specific therapy unanswered.[51]

\section{Treating Individuals}

Fortunately for clinicians treating these patients, there has been progress in identifying individuals for whom PFO-specific therapy (such as closure) might (at least in theory) be beneficial. The recently published RoPE score stratifies patients based on the probability an identified PFO is related to CS.[52] This CPM relies on clinical features that are associated with finding a PFO for an individual with CS and estimates an 'attributable fraction' for an identified PFO based on Bayes' theorem. Generally speaking for young patients without traditional stroke risk factors of hypertension or diabetes the likelihood an observed PFO is related to CS is high. Conversely, for an older patient with traditional stroke risk factors a PFO is likely to be incidental. Assuming that device closure does not help CS patients with incidental PFO, this therapy can be directed toward patients most likely to benefit however application of the CPM in this way has not yet been described. Despite our improved understanding of the contribution of PFO, important questions persist.

While the RoPE score estimates an 'attributable fraction' for an individual, this is a probability estimate and it remains unknown for a given individual whether an identified 
PFO was related to an index stroke. Stroke recurrence risk is highly variable and appear to be lowest for patients with the highest RoPE scores (most likely to have a PFO attributable stroke).[53] This raises important questions about the value of closure, especially in light of the occasional device related complications that are seen.[54] More work is needed to understand whether there are patients who have a high PFO attributable fraction and a reasonably high recurrence rate such that device based closure of PFO would offer clinical benefit.

\section{Conclusions}

CE stroke is caused by a variety of mechanisms and is associated with significant morbidity and risk of recurrence. The varied stroke mechanisms present important therapeutic targets for the treating clinicians. Despite our understandings of CE stroke mechanisms significant controversies remain regarding diagnostic testing and treatment decisions for individuals with CE strokes. Recent clinical trials and CPM development help to inform these debates though more work is needed to direct treatments to patients who are most likely to benefit.

\section{Acknowledgments}

Sources of Funding: National Institutes of Health (Grant Numbers: UL1TR001064; TL1 TR001062; R01 NS062153; R21 NS079826)

\section{References}

1. Lozano R, Naghavi M, Foreman K, Lim S, Shibuya K, Aboyans V, et al. Global and regional mortality from 235 causes of death for 20 age groups in 1990 and 2010: a systematic analysis for the Global Burden of Disease Study 2010. Lancet. 2012; 380:2095-128. [PubMed: 23245604]

2. Lackland DT, Roccella EJ, Deutsch AF, Fornage M, George MG, Howard G, et al. Factors influencing the decline in stroke mortality: a statement from the American Heart Association/ American Stroke Association. Stroke. 2014; 45:315-53. [PubMed: 24309587]

3. Go AS, Mozaffarian D, Roger VL, Benjamin EJ, Berry JD, Blaha MJ, et al. Heart disease and stroke statistics--2014 update: a report from the American Heart Association. Circulation. 2014:e28-e292. [PubMed: 24352519]

4. Arboix A, Alio J. Acute cardioembolic cerebral infarction: answers to clinical questions. Curr Cardiol Rev. 2012; 8:54-67. [PubMed: 22845816]

5. Pepi M, Evangelista A, Nihoyannopoulos P, Flachskampf Fa, Athanassopoulos G, Colonna P, et al. Recommendations for echocardiography use in the diagnosis and management of cardiac sources of embolism: European Association of Echocardiography (EAE) (a registered branch of the ESC). Eur J Echocardiogr. 2010; 11:461-76. [PubMed: 20702884]

6. Ferro JM. Cardioembolic stroke: an update. Lancet Neurol. 2003; 2:177-88. [PubMed: 12849239]

7. Jauch EC, Saver JL, Adams HP, Bruno A, Connors JJB, Demaerschalk BM, et al. Guidelines for the early management of patients with acute ischemic stroke: a guideline for healthcare professionals from the American Heart Association/American Stroke Association. Stroke. 2013; 44:870-947. [PubMed: 23370205]

8. Pepi M, Evangelista A, Nihoyannopoulos P, Flachskampf Fa, Athanassopoulos G, Colonna P, et al. Recommendations for echocardiography use in the diagnosis and management of cardiac sources of embolism: European Association of Echocardiography (EAE) (a registered branch of the ESC). Eur J Echocardiogr. 2010; 11:461-76. [PubMed: 20702884]

9. Morris JG, Duffis EJ, Fisher M. Cardiac workup of ischemic stroke: can we improve our diagnostic yield? Stroke. 2009; 40:2893-8. [PubMed: 19478214] 
10. Hahn RT, Abraham T, Adams MS, Bruce CJ, Glas KE, Lang RM, et al. Guidelines for performing a comprehensive transesophageal echocardiographic examination: recommendations from the american society of echocardiography and the society of cardiovascular anesthesiologists. J Am Soc Echocardiogr Elsevier Inc. 2013; 26:921-64.

11. Di Tullio MR. Patent foramen ovale: echocardiographic detection and clinical relevance in stroke. J Am Soc Echocardiogr American Society of Echocardiography. 2010; 23:144-55. quiz 220.

12. Wessler BS, Thaler DE, Ruthazer R, Weimar C, Di Tullio MR, Elkind MSV, et al. Transesophageal echocardiography in cryptogenic stroke and patent foramen ovale: analysis of putative high-risk features from the risk of paradoxical embolism database. Circ Cardiovasc Imaging. 2014; 7:125-31. [PubMed: 24214884]

13. Mojadidi MK, Roberts SC, Winoker JS, Romero J, Goodman-Meza D, Gevorgyan R, et al. Accuracy of transcranial Doppler for the diagnosis of intracardiac right-to-left shunt: a bivariate meta-analysis of prospective studies. JACC Cardiovasc Imaging Elsevier Inc. 2014; 7:236-50.

14. Serena J, Jiménez-Nieto M, Silva Y, Castellanos M. Patent foramen ovale in cerebral infarction. Curr Cardiol Rev. 2010; 6:162-74. [PubMed: 21804775]

15. Marini C, De Santis F, Sacco S, Russo T, Olivieri L, Totaro R, et al. Contribution of atrial fibrillation to incidence and outcome of ischemic stroke: results from a population-based study. Stroke. 2005; 36:1115-9. [PubMed: 15879330]

16. Hart RG, Pearce LA, Aguilar MI. Annals of Internal Medicine Review Meta-analysis: Antithrombotic Therapy to Prevent Stroke in Patients Who Have Nonvalvular Atrial Fibrillation. 2007

17. Flaker GC, Belew K, Beckman K, Vidaillet H, Kron J, Safford R, et al. Asymptomatic atrial fibrillation: demographic features and prognostic information from the Atrial Fibrillation Followup Investigation of Rhythm Management (AFFIRM) study. Am Heart J. 2005; 149:657-63. [PubMed: 15990749]

18. Healey JS, Connolly SJ, Gold MR, Israel CW, Van Gelder IC, Capucci A, et al. Subclinical atrial fibrillation and the risk of stroke. N Engl J Med. 2012; 366:120-9. [PubMed: 22236222]

19. Sanna T, Diener H-C, Passman RS, Di Lazzaro V, Bernstein Ra, Morillo Ca, et al. Cryptogenic stroke and underlying atrial fibrillation. N Engl J Med. 2014; 370:2478-86. [PubMed: 24963567]

20. Hindricks G, Pokushalov E, Urban L, Taborsky M, Kuck K-H, Lebedev D, et al. Performance of a new leadless implantable cardiac monitor in detecting and quantifying atrial fibrillation: Results of the XPECT trial. Circ Arrhythm Electrophysiol. 2010; 3:141-7. [PubMed: 20160169]

21. Gladstone DJ, Spring M, Dorian P, Panzov V, Thorpe KE, Hall J, et al. Atrial fibrillation in patients with cryptogenic stroke. N Engl J Med. 2014; 370:2467-77. [PubMed: 24963566]

22. Esperer HD, Esperer C, Cohen RJ. Cardiac arrhythmias imprint specific signatures on Lorenz plots. Ann Noninvasive Electrocardiol. 2008; 13:44-60. [PubMed: 18234006]

23. Gage BF, Waterman aD, Shannon W, Boechler M, Rich MW, Radford MJ. Validation of clinical classification schemes for predicting stroke: results from the National Registry of Atrial Fibrillation. JAMA. 2001; 285:2864-70. [PubMed: 11401607]

24. Lopes RD, Crowley MJ, Shah BR, Melloni C, Wood KA, Chatterjee R, Povsic TJ, Dupre ME, Kong DF, Barros e Silva PGM, Santos MHH, Armaganijan LV, Katz M, Kosinski A, McBroom AJ, Chobot MM, Gray R, Sanders GD. Stroke Prevention in Atrial Fibrillation. Compara.

25. January CT, Wann LS, Alpert JS, Calkins H, Cleveland JC, Cigarroa JE, et al. 2014 AHA/ACC/HRS Guideline for the Management of Patients With Atrial Fibrillation: A Report of the American College of Cardiology/American Heart Association Task Force on Practice Guidelines and the Heart Rhythm Society. Circulation. 2014

26. Lip GYH, Nieuwlaat R, Pisters R, Lane Da, Crijns HJGM. Refining clinical risk stratification for predicting stroke and thromboembolism in atrial fibrillation using a novel risk factor-based approach: the euro heart survey on atrial fibrillation. Chest. 2010; 137:263-72. [PubMed: 19762550]

27. Mason PK, Lake DE, DiMarco JP, Ferguson JD, Mangrum JM, Bilchick K, et al. Impact of the CHA2DS2-VASc score on anticoagulation recommendations for atrial fibrillation. Am J Med Elsevier Inc. 2012; 125:603.e1-6. 
28. Lloyd-Jones DM. Cardiovascular risk prediction: basic concepts, current status, and future directions. Circulation. 2010; 121:1768-77. [PubMed: 20404268]

29. Cook NR. Use and misuse of the receiver operating characteristic curve in risk prediction. Circulation. 2007; 115:928-35. [PubMed: 17309939]

30. Bajaj NS, Parashar A, Agarwal S, Sodhi N, Poddar KL, Garg A, et al. Percutaneous left atrial appendage occlusion for stroke prophylaxis in nonvalvular atrial fibrillation: a systematic review and analysis of observational studies. JACC Cardiovasc Interv. 2014; 7:296-304. [PubMed: 24650403]

31. Connolly SJ, Ezekowitz MD, Yusuf S, Eikelboom J, Oldgren J, Parekh A, et al. Dabigatran versus warfarin in patients with atrial fibrillation. N Engl J Med. 2009; 361:1139-51. [PubMed: 19717844]

32. Patel MR, Mahaffey KW, Garg J, Pan G, Singer DE, Hacke W, et al. Rivaroxaban versus warfarin in nonvalvular atrial fibrillation. N Engl J Med. 2011; 365:883-91. [PubMed: 21830957]

33. Granger CB, Alexander JH, McMurray JJV, Lopes RD, Hylek EM, Hanna M, et al. Apixaban versus warfarin in patients with atrial fibrillation. N Engl J Med. 2011; 365:981-92. [PubMed: 21870978]

34. Ruff CT, Giugliano RP, Braunwald E, Hoffman EB, Deenadayalu N, Ezekowitz MD, et al. Comparison of the efficacy and safety of new oral anticoagulants with warfarin in patients with atrial fibrillation: a meta-analysis of randomised trials. Lancet. 2014; 383:955-62. [PubMed: 24315724]

35. Giugliano RP, Ruff CT, Braunwald E, Murphy SA, Wiviott SD, Halperin JL, et al. Edoxaban versus warfarin in patients with atrial fibrillation. N Engl J Med. 2013; 369:2093-104. [PubMed: 24251359]

36. Douxfils J, Buckinx F, Mullier F, Minet V, Rabenda V, Reginster J-Y, et al. Dabigatran etexilate and risk of myocardial infarction, other cardiovascular events, major bleeding, and all-cause mortality: a systematic review and meta-analysis of randomized controlled trials. J Am Heart Assoc. 2014; 3:e000515. [PubMed: 24906369]

37. Patel MR, Hellkamp AS, Lokhnygina Y, Piccini JP, Zhang Z, Mohanty S, et al. Outcomes of discontinuing rivaroxaban compared with warfarin in patients with nonvalvular atrial fibrillation: analysis from the ROCKET AF trial (Rivaroxaban Once-Daily, Oral, Direct Factor Xa Inhibition Compared With Vitamin K Antagonism for Prevention o. J Am Coll Cardiol Elsevier Inc. 2013; 61:651-8.

38. Liakoni E, Rätz Bravo AE, Terracciano L, Heim M, Krähenbühl S. Symptomatic Hepatocellular Liver Injury With Hyperbilirubinemia in Two Patients Treated With Rivaroxaban. JAMA Intern Med. 2014

39. Lu G, DeGuzman FR, Hollenbach SJ, Karbarz MJ, Abe K, Lee G, et al. A specific antidote for reversal of anticoagulation by direct and indirect inhibitors of coagulation factor Xa. Nat Med. 2013; 19:446-51. [PubMed: 23455714]

40. Harrington AR, Armstrong EP, Nolan PE, Malone DC. Cost-effectiveness of apixaban, dabigatran, rivaroxaban, and warfarin for stroke prevention in atrial fibrillation. Stroke. 2013; 44:1676-81. [PubMed: 23549134]

41. Lechat P, Mas JL, Lascault G, Loron P, Theard M, Klimczac M, et al. Prevalence of patent foramen ovale in patients with stroke. N Engl J Med. 1988; 318:1148-52. [PubMed: 3362165]

42. Kutty S, Sengupta PP, Khandheria BK. Patent Foramen Ovale. J Am Coll Cardiol Elsevier Inc. 2012; 59:1665-71.

43. Alsheikh-Ali, Aa; Thaler, DE.; Kent, DM. Patent foramen ovale in cryptogenic stroke: incidental or pathogenic? Stroke. 2009; 40:2349-55. [PubMed: 19443800]

44. Meier B, Kalesan B, Mattle HP, Khattab AA, Hildick-Smith D, Dudek D, et al. Percutaneous closure of patent foramen ovale in cryptogenic embolism. N Engl J Med. 2013; 368:1083-91. [PubMed: 23514285]

45. Carroll JD, Saver JL, Thaler DE, Smalling RW, Berry S, MacDonald LA, et al. Closure of patent foramen ovale versus medical therapy after cryptogenic stroke. N Engl J Med. 2013; 368:1092100. [PubMed: 23514286] 
46. Furlan AJ, Reisman M, Massaro J, Mauri L, Adams H, Albers GW, et al. Closure or medical therapy for cryptogenic stroke with patent foramen ovale. N Engl J Med. 2012; 366:991-9. [PubMed: 22417252]

47. Kitsios GD, Thaler DE, Kent DM. Potentially large yet uncertain benefits: a meta-analysis of patent foramen ovale closure trials. Stroke. 2013; 44:2640-3. [PubMed: 23868270]

48. Messé SR, Kent DM. Still no closure on the question of PFO closure. N Engl J Med. 2013; 368:1152-3. [PubMed: 23514293]

49. Kernan WN, Ovbiagele B, Black HR, Bravata DM, Chimowitz MI, Ezekowitz MD, et al. Guidelines for the prevention of stroke in patients with stroke and transient ischemic attack: a guideline for healthcare professionals from the american heart association/american stroke association. Stroke. 2014:2160-236. [PubMed: 24788967]

50. Diener, H-C., et al. Rationale, objectives and design of a secondary stroke prevention study of dabigatran etexilate versus acetylsalicylic acid in patients with embolic stroke of undetermined source (RE-SPECT-ESUS). Presented at the European Stroke Confere;

51. Hart RG, Diener H-C, Coutts SB, Easton JD, Granger CB, O’Donnell MJ, et al. Embolic strokes of undetermined source: the case for a new clinical construct. Lancet Neurol. 2014; 13:429-38. [PubMed: 24646875]

52. Kent DM, Ruthazer R, Weimar C, Mas J-L, Serena J, Homma S, et al. An index to identify strokerelated vs incidental patent foramen ovale in cryptogenic stroke. Neurology. 2013; 81:619-25. [PubMed: 23864310]

53. Thaler DE, Ruthazer R, Weimar C, Mas J-L, Serena J, Di Angelantonio E, et al. Recurrent stroke predictors differ in medically treated patients with pathogenic vs other PFOs. Neurology. 2014; 83:221-6. [PubMed: 24928123]

54. Tobis J, Shenoda M. Percutaneous treatment of patent foramen ovale and atrial septal defects. J Am Coll Cardiol. 2012; 60:1722-32. [PubMed: 23040567] 


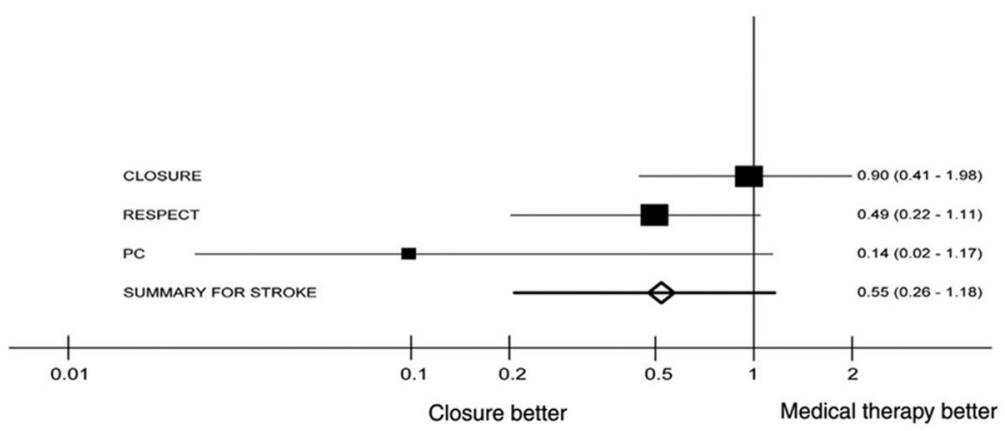

Figure 1.

Forest plot for the meta-analysis of hazards ratios of stroke of mechanical closure vs medical treatment from 3 randomized clinical clinical trials. 


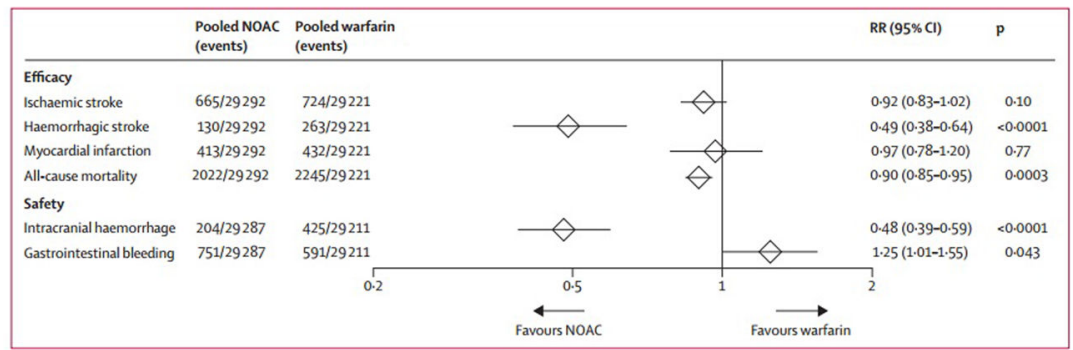

Figure 2. Secondary efficacy and safety outcomes

Data are $\mathrm{n} / \mathrm{N}$, unless otherwise indicated. Heterogeneity: ischaemic stroke $\mathrm{I}^{2}=32 \%, \mathrm{p}=0.22$; haemorrhagic stroke $\mathrm{I}^{2}=34 \%, \mathrm{p}=0.21$; myocardial infarction $\mathrm{I}^{2}=48 \%, \mathrm{p}=0.13$; all-cause mortality $\mathrm{I}^{2}=0 \%, \mathrm{p}=0.81$; intracranial haemorrhage $\mathrm{I}^{2}=32 \%, \mathrm{p}=0.22$; gastrointestinal bleeding $\mathrm{I}^{2}=74 \%, \mathrm{p}=0.009$. NOAC $=$ new oral anticoagulant. $\mathrm{RR}=$ risk ratio. 